\title{
Significance of Expression of IMP3, PPARG \& PD-L1 in Urothelial Carcinoma (Immunohistochemical Study)
}

\author{
Rasha M. El Sawi ${ }^{\text {a }}$, Ebtehal Abdel Aal ${ }^{\mathrm{a}}$, Amr El-Sebaaie ${ }^{\mathrm{b}}$, Nehal Zafer ${ }^{\mathrm{a}}$, Hala Agina ${ }^{\mathrm{a}}$
}

a Department of pathology, Benha faculty of medicine, Benha University, Egypt. b Department of pathology Military Medical Academy, cairo, Egypt

Correspondence to: Ebtehal AbdelAal, Department of pathology, Benha faculty of medicine, Benha University, Egypt.

\section{Email:}

ma_lolla@yahoo.com

Received: 8 December 2020

Accepted:13 December 2020

\begin{abstract}
:
Background: IMP3, PPARG and PD-L1 have been implicated in
\end{abstract} tumorigenesis and progression in many cancers, but their significance in urothelial carcinoma remains unclear. Aim: The aim of this study is to assess the possible significance of IMP3, PPARG and PD-L1 in urothelial bladder carcinoma and determine the relation between their expressions and different clinicopathological (tumor size, grade, histological variant, muscle invasion, lymphovascular invasion and pathologic $\mathrm{T}$ stage) and molecular variables. Methods: This study aimed to evaluate immunohistochemical expression of IMP3, PPARG and PD-L1 and correlate them with clinicopathological and molecular variables. Results: IMP3 was significantly correlated to muscle invasion $(\mathrm{P}<0.01)$, tumor grade $(\mathrm{P}<0.05)$, histological variant $(\mathrm{P}<0.05)$, lymphovascular invasion $(\mathrm{P}<0.01)$ and $\mathrm{PT}$ stage $(\mathrm{P}<0.01)$. PPARG was inversely correlated to muscle invasion $(\mathrm{P}<0.01)$, tumor grade $(\mathrm{P}<0.01)$, histological variant $(\mathrm{P}<0.01)$, PT stage $(\mathrm{P}<0.05)$ and molecular subtype $(\mathrm{P}<0.01)$. PD-L1 was significantly correlated to muscle invasion $(\mathrm{P}<0.05)$, histological variant $(\mathrm{P}<0.05)$ and $\mathrm{PT}$ stage $(\mathrm{P}<0.01)$. IMP3 and PD-L1 showed high statistically significant correlation $(\mathrm{P}<0.01)$. IMP3 and PPARG showed inverse statistically significant correlation $(\mathrm{P}<0.05)$. No significant correlation between PPARG and PD-L1expression was detected (P>0.05).Conclusions: IMP3 may be used as a prognostic marker. PPARG may be used to identify the luminal molecular subtype. PD-L1 may be used as a prognostic marker for tumor aggressiveness, so it may be used in immunotherapy.

Keyword: Urothelial carcinoma, IMP3, PPARG, PD-L1. 

Abbreviations: Urothelial bladder carcinoma (UBC), Insulin like growth factor II mRNA binding protein (IGF2BP3 / IMP3), Peroxisome Proliferator-Activated Receptor Gamma (PPAR $\gamma$ or PPARG), Programmed death ligand-1 (PD-L1).

\section{Introduction}

Bladder cancer $(\mathrm{BC})$ is the ninth most common diagnosed cancer worldwide and thirteenth most common cause of cancer death (1). Among BCs, urothelial cell carcinomas (UC) are the most common (2). It is the 5th most common cancer in men and the 12th most common cancer in women (3). In Egypt, urinary bladder cancer is the third most common cancer in both sexes representing $6.94 \%$ of total malignancies (4).

Smoking is the most important risk factor for UBC (5), followed by environmental pollution and occupational exposure to carcinogens namely, aromatic amines and polycyclic hydrocarbons (6).

Bladder cancers are categorized into two subtypes based on their microscopic findings into: non-muscle-invasive bladder cancer (NMIBC) and muscle invasive bladder cancer (MIBC) (7). Molecular subtyping identified "luminal" subtype that comprises the majority of early stage (non-invasive) bladder cancers and has better prognosis. On the other hand, the "basal" subtype is biologically aggressive and found almost exclusively in invasive tumors (8).

Clinicopathological parameters play a major role in determining the management of UBC, but are usually not reliable predictors of prognosis (9). Therefore, studying novel biomarkers of urothelial cancers, which have the potential value of serving as prognostic markers and new therapeutic targets, may solve this problem (10).

Insulin like growth factor II mRNA binding protein (IGF2BP3 / IMP3) is a member of the family of RNA-binding proteins which can bind RNAs strongly and specifically affecting their transcript target's fate (11). IMP3 is over-expressed in many human cancers, including lung cancer (12), gastric cancer (13), colon cancer (14), and pancreatic cancer (15).

Another marker of interest is peroxisome proliferator-activated receptor gamma (PPAR $\gamma$ / PPARG), a member of the nuclear receptor superfamily of transcription factors that functions as a master regulator of adipocyte differentiation and metabolism (16). Its 
biological significance in cancer still remains controversial. Activation of PPARG can induce either tumor suppressive or promoting responses (17).

Programmed death ligand-1 (PD-L1) is a type I transmembrane glycoprotein that plays an important role in immune suppression (18). Tumor cells often use the PD-1/PD-L1 pathway to protect themselves from the tumor-specific $\mathrm{T}$ cells (19). Blockade of the immune checkpoints activated by the PD-1/PD-L1 pathway has demonstrated impressive benefits in melanoma, hematologic malignancies and numerous solid tumors such as non-small cell lung cancer (20).

This study aimed to evaluate immunohistochemical expression of IMP3, PPARG and PD-L1 and correlate them with clinicopathological and molecular variables of urothelial bladder carcinoma.

\section{Subjects and Methods:}

This retrospective study was carried upon 45 selected cases of urothelial bladder carcinoma with 6 control cases of normal urothelium. The studied cases included archival formalin-fixed, paraffin embedded blocks processed from January 2015 to December 2019 from the Pathology Department of Benha Faculty of Medicine and the International Medical Center Hospital, Cairo, Egypt, after obtaining approval from the institutional ethics committee. Twelve cases were obtained by radical cystectomy and 33 cases by transurethral resection of the bladder tumour (TURBT). All sections were reviewed for confirmation of the original diagnosis. Tumour grade and stage were based on pathologic findings following the World Health Organization grading system (WHO 2016) and the American Joint Committee on Cancer staging manual, 8th edition, respectively (21) and (22). Molecular subtyping were obtained from the patient archives based on previous immunostaining of combined GATA3 and CK5/6 that classified the cases into: luminal subtype (GATA3+ve, CK5/6-ve), basal subtype (GATA3-ve, CK5/6+ve) and double negative subtype (GATA3-ve, CK5/6-ve) (23).

Histopathological evaluation: hematoxylin and eosin-stained slides on all cases were reviewed to classify the lesions into 24 non-muscle invasive bladder cancers (NMIBC) and 21 muscle invasive bladder cancers (MIBC) (7).

Immunohistochemical evaluation: Four micron tissue sections were obtained from formalin-fixed, paraffin-embedded tissue 
blocks on coated slides. The manufacturer's instructions were followed using a standard labelled streptavidinbiotin system (DakoCytomation A/S, Glostrup, Denmark). Antigen retrieval was performed by using $10 \mathrm{mmol} / \mathrm{L}$ citrate monohydrate buffer $(\mathrm{pH}$ 6.0) and heated for 15 minutes in the microwave.

The slides then were immunostained for IMP3 Rabbit polyclonal antibody $(0.1 \mathrm{mg} / \mathrm{ml}$ concentration, Chongqing Biospes Company, Cat No YPA1463, China) at a dilution of 1:100 and PPARG Rabbit polyclonal antibody (Chongqing Biospes Company, Cat No YPA1724, China) at a dilution of 1:50 and slides for both markers were incubated at $4^{\circ} \mathrm{C}$ overnight. Immunoreaction was visualized by adding $\mathrm{DAB}$ as a chromagen. Fetal liver tissue obtained from aborted fetus (16 weeks) was used as a positive control for IMP3 (24). Breast duct carcinoma tissue was used as a positive control for PPARG (25). For negative controls, the primary antibodies were omitted from the staining procedure.

For PD-L1, mouse monoclonal antibody (clone 22C3 pharmDx, Dako, ready to use, Denmark) was assessed with the Omnis Autostainer using the optimized closed protocol for the automated platform passing through the previous steps in an automated manner. The (NCIH226) was used as a PD-L1 positive control cell line and (MCF-7) was used as a PD-L1 negative control cell line (26).

- Immunohistochemical assessment: -Assessment of IMP3 expression: IMP3 expression was detected as cytoplasmic brownish staining of tumour cells. It was evaluated using a 4-tiered system for staining intensity: Score 0 was assigned if cells were completely negative, score $1+$ if at least $10 \%$ of cells stained weakly, score $2+$ if at least $10 \%$ of cells stained moderately, and score $3+$ for strong cytoplasmic staining in $>10 \%$ of cells (27).

-Assessment of PPARG expression:

The PPARG was detected as nuclear brown coloration. The average of positive cells was determined by analysing 5 randomized fields containing 200 cells per field and interpreted as follows: Tumours expressing less than $10 \%$ were defined as negative, low positive if between $10 \%$ and $40 \%$ expression and high positive if more than $40 \%$ expression. For statistical analysis, low and high positives were considered positive (28). 
-Assessment of PD-L1 expression:

According to the PD-L1 expression assessment recommendations, positive membrane staining, irrespective of its intensity, was evaluated in tumour cells (TC) and tumour-infiltrating mononuclear immune cells (IC). The cut off for the proportions of stained cells in a positive sample was considered as $\mathrm{TC} \pm$ IC $\geq 10 \%$ as recommended by US FDA $(29,30)$.

Statistical analysis: Results were analyzed using SPSS (version 20) (Chicago, Ill, USA). The Pearson correlation coefficient was used for statistical analysis. $\mathrm{P}$ value $<0.05$ was considered statistically significant and highly statistically significant when it was $<0.01$. Receiver-operating characteristic (ROC) curve was used to predict sensitivity, specificity and accuracy of PPARG and PD-L1.

\section{Results:}

The studied 45 urothelial carcinoma cases were classified as; 24 cases $(53.3 \%)$ nonmuscle invasive bladder cancer (NMIBC) and 21 cases $(46.7 \%)$ muscle invasive bladder cancer (MIBC). They were graded into: 18 cases (40\%) of low grade and 27 cases $(60 \%)$ of high grade urothelial carcinoma. According to the histological variant, cases were classified into: 25 cases $(55.6 \%)$ were papillary urothelial carcinomas and 20 cases $(44.4 \%)$ were non-papillary (solid, nested and micropapillary). Out of 45 studied cases, 12 cases $(26.7 \%)$ were PTa, 12 cases (26.7\%) were PT1, 16 cases $(35.6 \%)$ were PT2, 3 cases $(6.6 \%)$ were PT3 and 2 cases (4.4\%) were PT4. Seven cases (15.6\%) were positive for lymphovascular invasion and 38 cases $(84.4 \%)$ were negative. Prior IHC staining by GATA3 and CK 5/6 antibodies for the cases categorized them as 25 cases $(55.6 \%)$ were luminal, 14 cases $(31.1 \%)$ were basal and 6 cases $(13.3 \%)$ were double negative.

\section{Immunohistochemical Results:}

Out of 45 cases of UBC, IMP3 expression was detected in the cytoplasm of $(88.9 \%)$ of urothelial carcinoma cases. Five cases $(11.1 \%)$ were score 0,18 cases $(40 \%)$ were score $1+, 14$ cases $(31.1 \%)$ were score $2+$ and 8 cases $(17.8 \%)$ were score $3+$ (figure 1). The normal control cases of urothelium were negative for IMP3. A high statistically significant correlation between IMP3 expression and muscle invasion, LVI and PT stage was found (P $<0.01$ for all. A statistical significant correlation with tumour grade and 
histological variant was present $(\mathrm{P}<0.05$ for both). Insignificant correlation with tumour size and molecular subtype was found $(\mathrm{P}>0.05)$.

PPARG immunostaining showed nuclear expression of the malignant cells (figure 2). The control normal urothelium also expressed uniform nuclear stain. PPARG expression was statistically significant and inversely associated with muscle invasion $(\mathrm{P}<0.01)$, high tumour grade $(\mathrm{P}<0.01)$, non-papillary histological variant $(\mathrm{P}<0.01)$, high PT stage $(\mathrm{P}<0.05)$ and non-luminal molecular subtype $(\mathrm{P}<0.01)$. It was statistically insignificant with tumour size and LVI $(\mathrm{P}>0.05)$.

PD-L1 IHC was expressed in tumour cell membrane and tumour infiltrating mononuclear cells (TIMCs). Positive expression for PD-L1 was detected in (24.4\%) of our cases and (75.6\%) were negative (figure 3). The control normal urothelium did not express PD-L1. The expression of PD-L1 in muscle-invasive tumours was significantly higher than that in non-muscle-invasive $(\mathrm{P}<0.05)$. There was a statistically significant correlation between PD-L1 expression and both histological variant $(\mathrm{P}<0.05)$ and $\mathrm{PT}$ stage $(\mathrm{P}<0.01)$. It was statistically insignificant with tumour size, grade, LVI and molecular subtype $(\mathrm{P}>0.05)$.

According to ROC curve, area under the curve (AUC) of PPARG was 0.87 (very good).The sensitivity; specificity and accuracy of PPARG expression in the diagnosis of molecular subtype of urothelial carcinoma were 92\%, 65\% and $80 \%$ respectively. According to ROC curve, area under the curve (AUC) of PDL1 was 0.91 (Excellent). The sensitivity, specificity and accuracy of PPARG expression in the prediction of Pathologic $\mathrm{T}$ stage of urothelial carcinoma were $100 \%, 85 \%$ and $86.7 \%$ respectively (figure 4).

Correlation between the studied immunohistochemical expressions of IMP3, PPARG and PD-L1 showed: High statistically significant correlation between IMP3 and PD-L1 $(\mathrm{P}<0.01)$, Inverse statistically significant correlation between IMP3 and PPARG $(\mathrm{P}<0.05)$ and Insignificant correlation between PPARG and PD-L1(P > 0.05).

The results of IMP3, PPARG and PD-L1 antibodies were correlated to different clinicopathological and molecular variables and summarized in tables 1,2 and 3 . 

Table (1): Relation between IMP3 expression and clinicopathological \& molecular variables:

\begin{tabular}{|c|c|c|c|c|c|c|c|c|c|c|c|}
\hline \multirow{2}{*}{\multicolumn{2}{|c|}{ Variable }} & \multirow{4}{*}{$\begin{array}{l}\text { Total } \\
21\end{array}$} & \multicolumn{8}{|c|}{ IMP3 expression } & \multirow{3}{*}{$P$ value } \\
\hline & & & \multicolumn{2}{|c|}{ Score 0} & \multicolumn{2}{|c|}{ Score 1+ } & \multicolumn{2}{|c|}{ Score 2+ } & \multicolumn{2}{|c|}{ Score 3+ } & \\
\hline & & & No. & $\%$ & NO. & $\%$ & NO. & $\%$ & NO. & & \\
\hline \multirow[t]{2}{*}{ Tumour size } & $<3 \mathrm{~cm}$ & & 2 & $9.5 \%$ & 9 & $42.9 \%$ & 5 & $23.8 \%$ & 5 & $23.8 \%$ & \multirow[t]{2}{*}{$>0.05$} \\
\hline & $\geq 3 \mathrm{~cm}$ & 24 & 3 & $12.5 \%$ & 9 & $37.5 \%$ & 9 & $37.5 \%$ & 3 & $12.5 \%$ & \\
\hline \multirow{4}{*}{$\begin{array}{l}\text { Muscle } \\
\text { invasion } \\
\text { Grade }\end{array}$} & NMIBC & 24 & 4 & $16.7 \%$ & 12 & $50 \%$ & 7 & $29.2 \%$ & 1 & $4.1 \%$ & \multirow[t]{2}{*}{$<0.01 * *$} \\
\hline & MIBC & 21 & 1 & $4.8 \%$ & 6 & $28.6 \%$ & 7 & $33.3 \%$ & 7 & $33.3 \%$ & \\
\hline & Low grade & 18 & 3 & $16.7 \%$ & 9 & $50 \%$ & 5 & $27.8 \%$ & 1 & $5.6 \%$ & \multirow[t]{2}{*}{$<0.05^{*}$} \\
\hline & High grade & 27 & 2 & $7.4 \%$ & 9 & $33.3 \%$ & 9 & $33.3 \%$ & 7 & $26 \%$ & \\
\hline \multirow{7}{*}{$\begin{array}{l}\text { Histological } \\
\text { variant } \\
\text { Pathologic T } \\
\text { stage }\end{array}$} & Papillary & 25 & 3 & $12 \%$ & 12 & $48 \%$ & 8 & $32 \%$ & 2 & $8 \%$ & \multirow[t]{2}{*}{$<0.05^{*}$} \\
\hline & Non-papillary & 20 & 2 & $10 \%$ & 6 & $30 \%$ & 6 & $30 \%$ & 6 & $30 \%$ & \\
\hline & PTa & 12 & 2 & $16.7 \%$ & 5 & $41.7 \%$ & 4 & $33.3 \%$ & 1 & $8.3 \%$ & \multirow[t]{5}{*}{$<0.01 * *$} \\
\hline & PT1 & 12 & 2 & $16.7 \%$ & 7 & $58.3 \%$ & 3 & $25 \%$ & 0 & $0 \%$ & \\
\hline & PT2 & 16 & 1 & $6.2 \%$ & 6 & $37.5 \%$ & 5 & $31.2 \%$ & 4 & $25 \%$ & \\
\hline & PT3 & 3 & 0 & $0 \%$ & 0 & $0 \%$ & 2 & $66.7 \%$ & 1 & $33.3 \%$ & \\
\hline & PT4 & 2 & 0 & $0 \%$ & 0 & $0 \%$ & 0 & $0 \%$ & 2 & $100 \%$ & \\
\hline \multirow[t]{2}{*}{ LVI } & $+\mathrm{ve}$ & 7 & 0 & $0 \%$ & 0 & $0 \%$ & 3 & $42.9 \%$ & 4 & $57.1 \%$ & \multirow[t]{2}{*}{$<0.01 * *$} \\
\hline & -ve & 38 & 5 & $13.2 \%$ & 18 & $47.4 \%$ & 11 & $28.9 \%$ & 4 & $10.5 \%$ & \\
\hline \multirow{3}{*}{ subtype } & Luminal & 25 & 4 & $16 \%$ & 13 & $52 \%$ & 7 & $28 \%$ & 1 & $4 \%$ & \multirow[t]{4}{*}{$>0.05$} \\
\hline & Basal & 14 & 1 & $7.1 \%$ & 4 & $28.6 \%$ & 5 & $35.7 \%$ & 4 & $28.6 \%$ & \\
\hline & Double-ve & 6 & 0 & $0 \%$ & 1 & $16.7 \%$ & 2 & $33.3 \%$ & 3 & $50 \%$ & \\
\hline Total & & 45 & 5 & $11.1 \%$ & 18 & $40 \%$ & 14 & $31.1 \%$ & 8 & $17.8 \%$ & \\
\hline
\end{tabular}

IMP3: Insulin like growth factor II mRNA binding protein, NMIBC: Non-Muscle Invasive Bladder Cancer

MIBC: Muscle Invasive Bladder Cancer, PT: Pathologic tumour, LVI: Lymphovascular invasion

*Significant **highly significant

Table (2): Relation between PPARG expression and clinicopathological \& molecular variables:

\begin{tabular}{|c|c|c|c|c|c|c|c|c|c|}
\hline & \multicolumn{9}{|c|}{ PPARG expression } \\
\hline & \multirow{3}{*}{ Feature } & \multirow[t]{3}{*}{ Total } & \multirow{2}{*}{\multicolumn{2}{|c|}{ Negative }} & \multicolumn{4}{|c|}{ Positive } & \multirow{3}{*}{$P$ value } \\
\hline & & & & & \multicolumn{2}{|c|}{ Low } & \multicolumn{2}{|c|}{ High } & \\
\hline & & & No. & $\%$ & NO. & $\%$ & No. & $\%$ & \\
\hline \multirow[t]{2}{*}{ Tumour size } & $<3 \mathrm{~cm}$ & 21 & 6 & $28.6 \%$ & 5 & $23.8 \%$ & 10 & $47.6 \%$ & $>0.05$ \\
\hline & $\geq 3 \mathrm{~cm}$ & 24 & 9 & $37.5 \%$ & 8 & $33.3 \%$ & 7 & $29.2 \%$ & \\
\hline \multirow[t]{2}{*}{ Muscle invasion } & NMIBC & 24 & 3 & $12.5 \%$ & 7 & $29.2 \%$ & 14 & $58.3 \%$ & $<0.01 * *$ \\
\hline & MIBC & 21 & 12 & $57.1 \%$ & 6 & $28.6 \%$ & 3 & $14.3 \%$ & \\
\hline \multirow[t]{2}{*}{ Grade } & Low grade & 18 & 1 & $5.6 \%$ & 6 & $33.3 \%$ & 11 & $61.1 \%$ & $<0.01 * *$ \\
\hline & High grade & 27 & 14 & $51.9 \%$ & 7 & $25.9 \%$ & 6 & $22.2 \%$ & \\
\hline \multirow[t]{2}{*}{ Histological variant } & Papillary & 25 & 4 & $16 \%$ & 7 & $28 \%$ & 14 & $56 \%$ & $<0.01 * *$ \\
\hline & Non-papillary & 20 & 11 & $55 \%$ & 6 & $30 \%$ & 3 & $15 \%$ & \\
\hline \multirow[t]{5}{*}{ Pathologic T stage } & PTa & 12 & 0 & $0 \%$ & 3 & $25 \%$ & 9 & $75 \%$ & $<0.05 *$ \\
\hline & PT1 & 12 & 3 & $25 \%$ & 4 & $33.3 \%$ & 5 & $41.7 \%$ & \\
\hline & PT2 & 16 & 10 & $62.5 \%$ & 3 & $18.8 \%$ & 3 & $18.8 \%$ & \\
\hline & PT3 & 3 & 1 & $33.3 \%$ & 2 & $66.7 \%$ & 0 & $0 \%$ & \\
\hline & PT4 & 2 & 1 & $50 \%$ & 1 & $50 \%$ & 0 & $0 \%$ & \\
\hline \multirow[t]{2}{*}{ LVI } & $+\mathrm{ve}$ & 7 & 3 & $42.9 \%$ & 3 & $42.9 \%$ & 1 & $14.3 \%$ & $>0.05$ \\
\hline & -ve & 38 & 12 & $31.6 \%$ & 10 & $26.3 \%$ & 16 & $42.1 \%$ & \\
\hline \multirow[t]{3}{*}{ Molecular subtype } & Luminal & 25 & 2 & $8 \%$ & 7 & $28 \%$ & 16 & $64 \%$ & $<0.01 * *$ \\
\hline & Basal & 14 & 9 & $64.3 \%$ & 5 & $35.7 \%$ & 0 & $0 \%$ & \\
\hline & Double-ve & 6 & 4 & $66.7 \%$ & 1 & $16.7 \%$ & 1 & $16.7 \%$ & \\
\hline Total & & 45 & 15 & $33.3 \%$ & 13 & $28.9 \%$ & 17 & $37.8 \%$ & \\
\hline
\end{tabular}

PPARG: Peroxisome Proliferator-Activated Receptor Gamma (PPAR $\gamma$ ), NMIBC: Non-Muscle Invasive Bladder Cancer, MIBC: Muscle Invasive Bladder Cancer, PT: Pathologic tumor, LVI: Lymphovascular invasion *Significant $\quad * *$ Highly significant 
Table (3): Relation between PD-L1 expression and clinicopathological \& molecular variables:

\begin{tabular}{|c|c|c|c|c|c|c|c|}
\hline \multirow{3}{*}{ 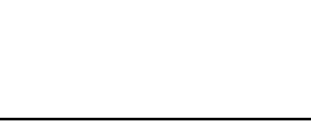 } & \multirow[t]{3}{*}{ Variable } & \multicolumn{5}{|c|}{ PD-L1 expression } & \multirow{3}{*}{$P$ value } \\
\hline & & \multirow[t]{2}{*}{ Total } & \multicolumn{2}{|c|}{ Negative } & \multicolumn{2}{|c|}{ Positive } & \\
\hline & & & No. & $\%$ & NO. & $\%$ & \\
\hline \multirow[t]{2}{*}{ Tumour size } & $<3 \mathrm{~cm}$ & 21 & 17 & $81 \%$ & 4 & $19 \%$ & $>0.05$ \\
\hline & $\geq 3 \mathrm{~cm}$ & 24 & 17 & $70.8 \%$ & 7 & $29.2 \%$ & \\
\hline \multirow{2}{*}{ Muscle invasion } & NMIBC & 24 & 21 & $87.5 \%$ & 3 & $12.5 \%$ & $<0.05^{*}$ \\
\hline & MIBC & 21 & 13 & $61.9 \%$ & 8 & $38.1 \%$ & \\
\hline \multirow[t]{2}{*}{ Grade } & Low grade & 18 & 16 & $88.9 \%$ & 2 & $11.1 \%$ & $>0.05$ \\
\hline & High grade & 27 & 18 & $66.7 \%$ & 9 & $33.3 \%$ & \\
\hline \multirow[t]{2}{*}{ Histological variant } & Papillary & 25 & 22 & $88 \%$ & 3 & $12 \%$ & $<0.05^{*}$ \\
\hline & Non-papillary & 20 & 12 & $60 \%$ & 8 & $40 \%$ & \\
\hline \multirow[t]{5}{*}{ Pathologic T stage } & PTa & 12 & 11 & $91.7 \%$ & 1 & $8.3 \%$ & $<0.01 * *$ \\
\hline & PT1 & 12 & 10 & $83.3 \%$ & 2 & $16.7 \%$ & \\
\hline & PT2 & 16 & 13 & $81.2 \%$ & 3 & $18.8 \%$ & \\
\hline & PT3 & 3 & 0 & $0 \%$ & 3 & $100 \%$ & \\
\hline & PT4 & 2 & 0 & $0 \%$ & 2 & $100 \%$ & \\
\hline \multirow[t]{2}{*}{ LVI } & $+v e$ & 7 & 5 & $71.4 \%$ & 2 & $28.6 \%$ & $>0.05$ \\
\hline & -ve & 38 & 29 & $76.3 \%$ & 9 & $23.7 \%$ & \\
\hline \multirow[t]{3}{*}{ Molecular subtype } & Luminal & 25 & 23 & $92 \%$ & 2 & $8 \%$ & $>0.05$ \\
\hline & Basal & 14 & 7 & $50 \%$ & 7 & $50 \%$ & \\
\hline & Double-ve & 6 & 4 & $66.7 \%$ & 2 & $33.3 \%$ & \\
\hline Total & & 45 & 34 & $75.6 \%$ & 11 & $24.4 \%$ & \\
\hline
\end{tabular}

PD-L1: Programmed death ligand-1, NMIBC: Non-Muscle Invasive Bladder Cancer, MIBC: Muscle Invasive Bladder Cancer, PT: Pathologic tumour, LVI: Lymphovascular invasion

*Significant $\quad * *$ highly significant

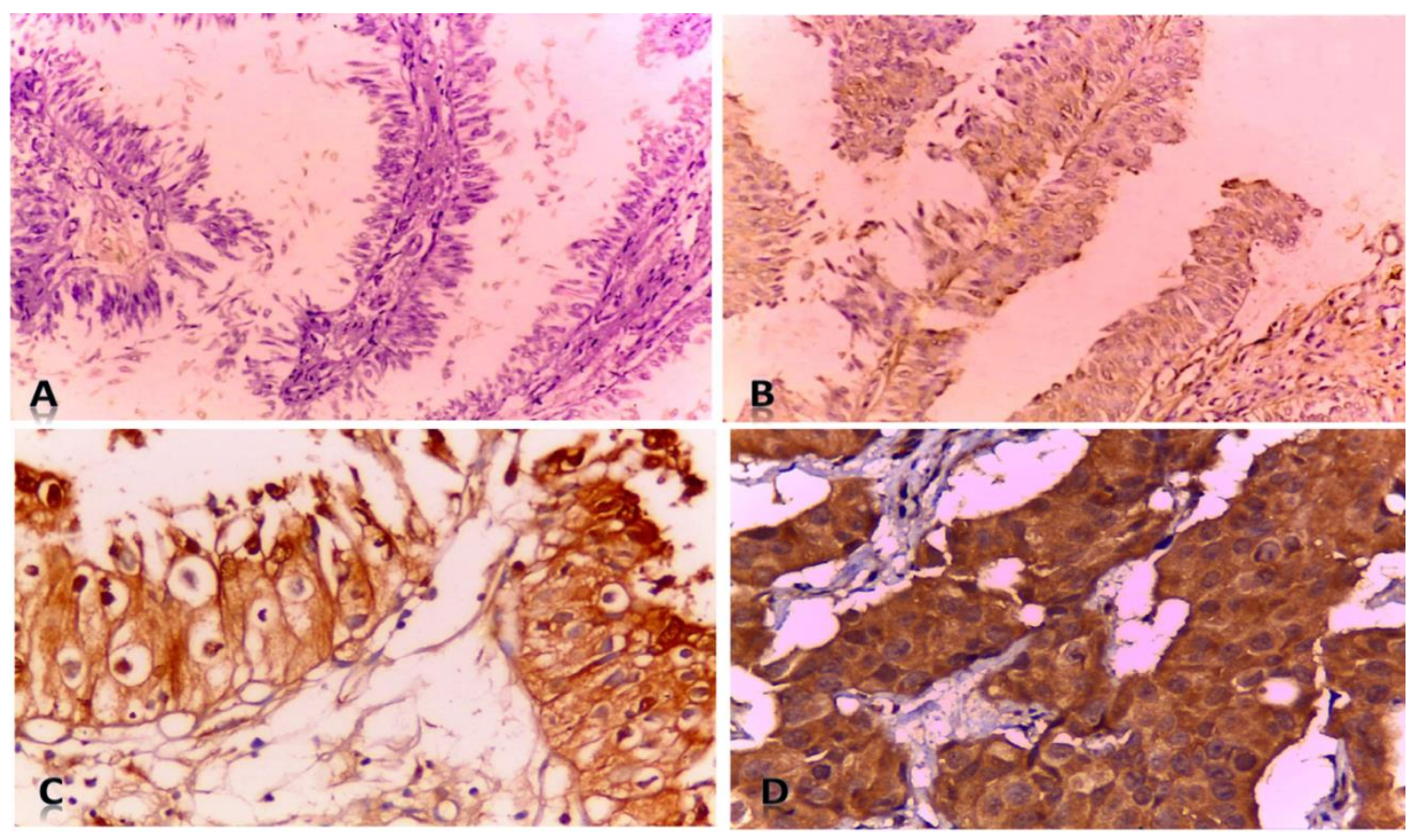

Figure (1): A: Score 0 for IMP3 immunohistochemistry without cytoplasmic staining in the tumor cells of a non-muscle invasive urothelial carcinoma, low grade, papillary variant (ABC x 200). B: Score 1+ for IMP3 immunohistochemistry showing mild cytoplasmic staining in at least $10 \%$ of cells of a non-muscle invasive urothelial carcinoma, low grade, papillary variant (ABC x 200). C: Score $2+$ for IMP3 immunohistochemistry showing moderate cytoplasmic staining in at least $10 \%$ of cells of a non-muscle invasive urothelial carcinoma, high grade, papillary variant (ABC x 400). D: Score 3+ for IMP3 immunohistochemistry showing strong cytoplasmic staining in $>10 \%$ of cells of a muscle invasive urothelial carcinoma, high grade, solid variant (ABC x 400). 


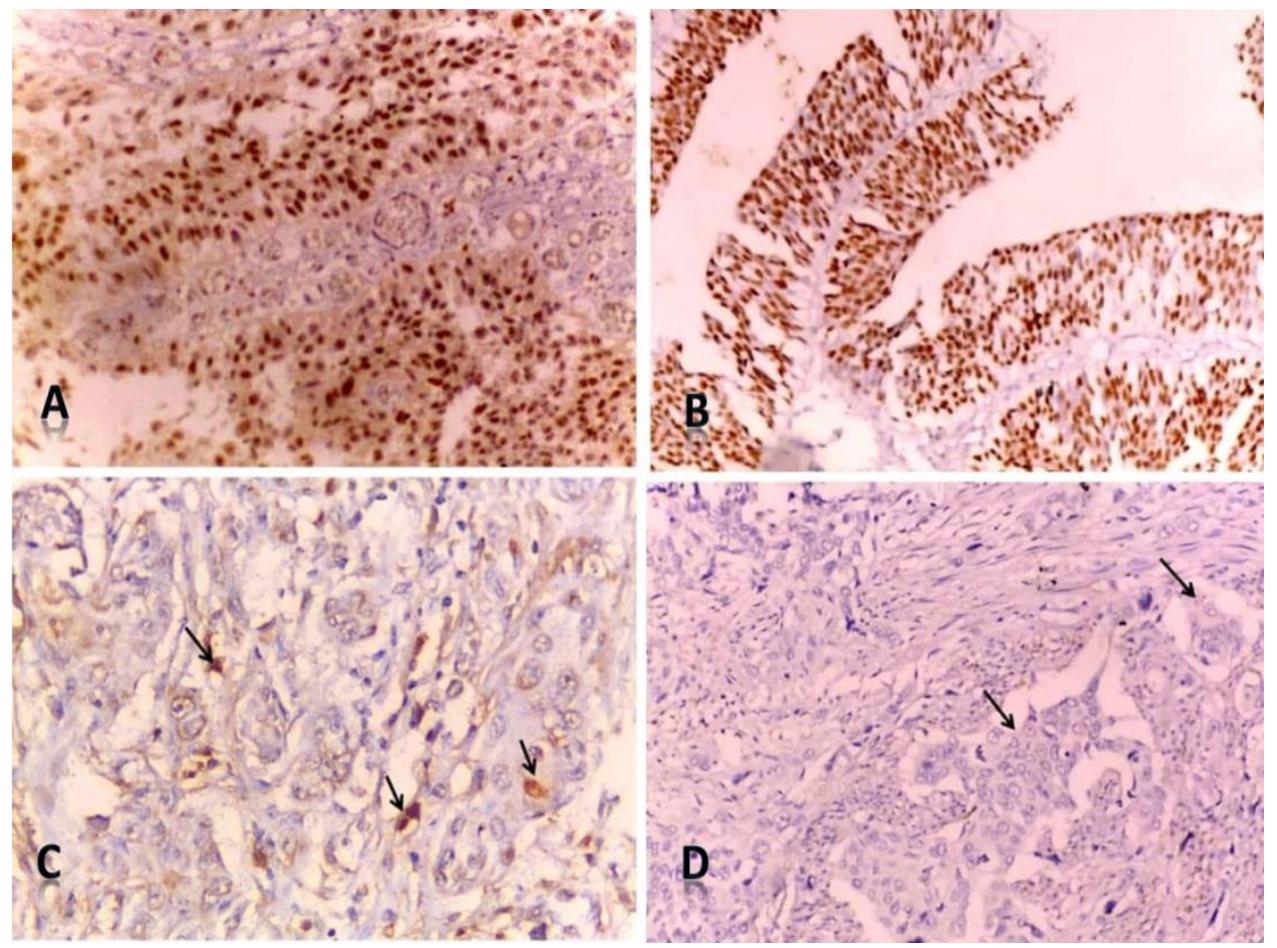

Figure (2): A, B: High positive expression for PPARG immunohistochemistry showing nuclear staining in $>40 \%$ of cells of a non-muscle invasive urothelial carcinoma, low grade, papillary variant ( $\mathrm{ABC} x$ 200). C: Low positive expression for PPARG immunohistochemistry showing nuclear staining in $>10 \%$, but $<40 \%$ of cells of a muscle invasive urothelial carcinoma, high grade, solid variant (arrows) (ABC x 400). D: Negative expression for PPARG immunohistochemistry showing no nuclear staining of a muscle invasive urothelial carcinoma, high grade, solid variant (ABC x 200).

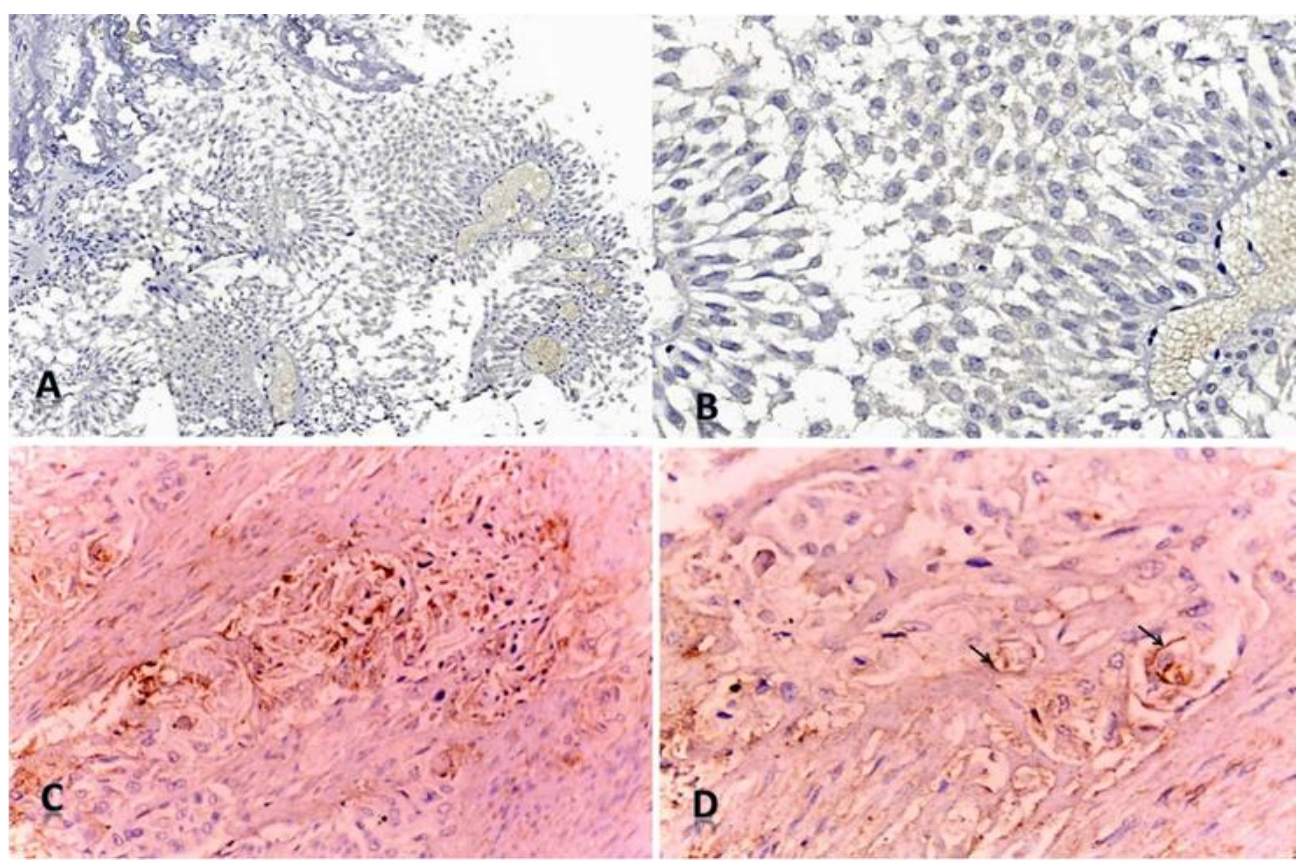

Figure (3): A,B: Negative expression for PD-L1 immunohistochemistry showing no membranous staining of a non-muscle invasive urothelial carcinoma, low grade, papillary variant ( $\mathrm{ABC} \times \mathrm{40}$ ) and (ABC x 400). C, D: Positive expression for PD-L1 immunohistochemistry showing membranous staining of TC \pm IC $\geq 10 \%\{C P S$ score $\geq 10 \%$ \} of a muscle invasive urothelial carcinoma, high grade, solid variant (ABC x 200) and (ABC $\mathrm{x}$ 400). 


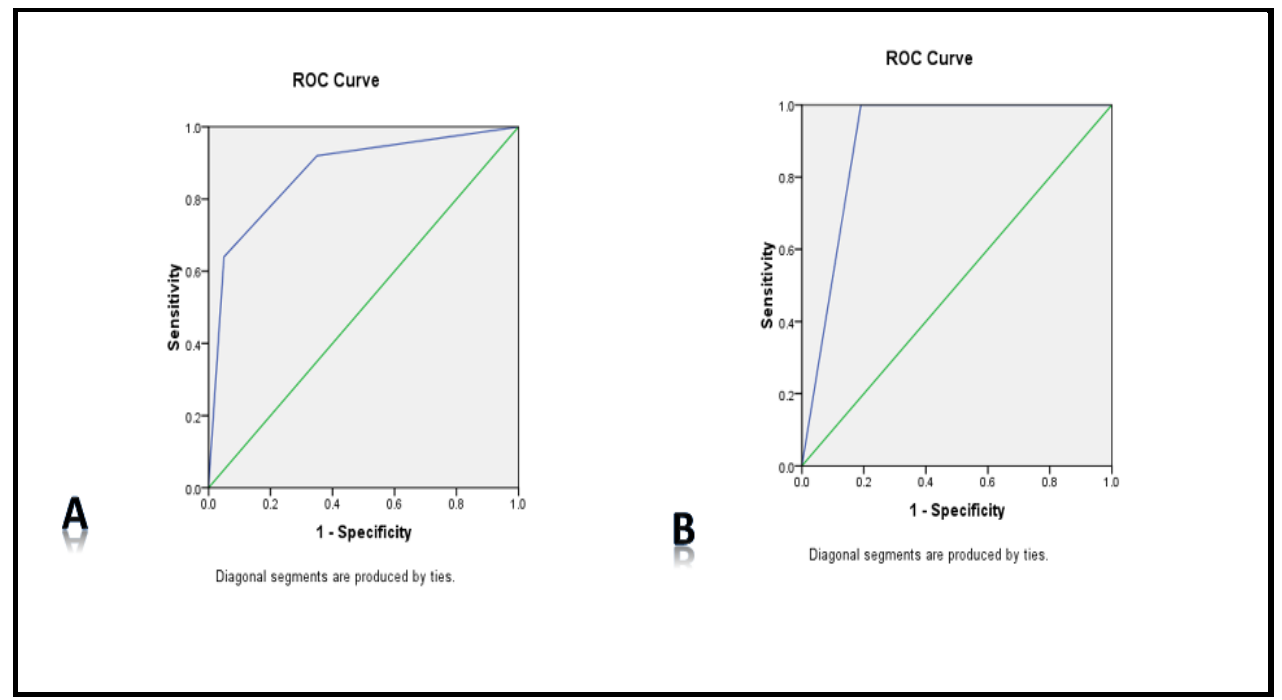

Figure 4: A: Validity of PPARG expression in diagnosis of molecular subtype. B: Validity of PD-L1 expression in prediction of Pathologic T stage.

\section{Discussion}

Studying novel biomarkers of urothelial carcinoma, that have the potential value of serving as prognostic markers and new therapeutic targets, is still a problem to be solved. In this study, there was a high statistically significant correlation between IMP3 expression and muscle invasion $(\mathrm{P}<0.01)$. This is consistent with the results which stated that the expression of IMP3 in muscle-invasive samples was significantly higher than that in non-muscle-invasive urothelial carcinoma specimens $\quad(\mathrm{P}=0.008)$ suggesting that IMP3 may identify patients with a high potential to develop invasive bladder cancer (31).

In this work, IMP3 expression was significantly related to the tumour grade $(\mathrm{P}<0.05)$. This is consistent with previously reported results stating that significant associations were found between IMP3 and advanced grade in urinary bladder cancer $(\mathrm{P}<0.0001)(\mathbf{3 2})$.

IMP3 expression in this study was highly statistically significant with the pathologic $\mathrm{T}$ stage $(\mathrm{P}<0.01)$. In parallel to this, in a study done on tumours including urothelial carcinoma, it was found that the expression of IMP3 is interestingly and significantly; related to the advanced stage $(\mathrm{P}<0.001)$ and to the aggressive behavior of tumours demonstrating the prognostic importance of IMP3 expression and that it may identify bladder cancer patients at high risk of progression (33). Disagreeing with that, it was found that IMP3 expression was not associated with the pathological features such as tumour grade $(\mathrm{P}=0.12)$ and stage 
$(\mathrm{P}=0.33)$. This may be due to racial and genetic differences as well as different antibody clones used and different number of cases (34).

There was a high statistical significant correlation between IMP3 expression and the lymphovascular invasion (LVI) in our study $(\mathrm{P}<0.01)$ matching with a study which found that IMP3 positivity was significantly related to LVI in their cases $(\mathrm{P}=0.006)(\mathbf{3 5})$.

In the present study, PPARG expression was highly statistically significant and inversely associated with the muscle invasion $(\mathrm{P}<0.01)$. This is consistent with study which reported an inverse significant correlation between PPARG expression and the muscle invasion status $(\mathrm{P}=0.004)$ (36). On the contrary, it was found that PPARG immunoexpression was elevated in invasive bladder cancers compared with the superficial tumors $(\mathrm{P}=0.002)$. This disagreement may be due to different primary antibodies used, different interpretation method and heterogeneity of urothelial bladder cancer (37).

In this study, there was a high statistically significant and inverse correlation between PPARG expression and the tumour grade $(\mathrm{P}<0.01)$. This is in a perfect concordance with another similar study where it was stated that in human urothelial cancer, low PPARG was significantly associated with poor prognosis factors, such as high histological grade $(\mathrm{p}=0.0160)$ (28). Disagreeing with that, it was found that PPARG expression was higher in $\mathrm{G} 3$ of bladder cancer than in G1 $(\mathrm{P}<0.001) \quad$ (38). This may be attributable to that the goat polyclonal antibodies (PPAR- $\alpha, \beta$ and $\gamma$ ) used in their study differ from our rabbit antibody and may be due to the different interpretation method.

As regard the pathologic $\mathrm{T}$ stage in this study, there was a statistically significant correlation between PPARG expression and the PT stage $(\mathrm{P}<0.05)$. Agreeing with what was found that PPARG was immunodetected more frequently in the superficial $(\mathrm{Ta}-\mathrm{T} 1)$ than the invasive $(\mathrm{T} 2-\mathrm{T} 4)$ tumours $(\mathrm{P}<0.001)$ (25). These findings suggest that PPARG expression can identify patients with a better prognosis. Unlikely, it was found that PPARG expression was higher in advanced cancer (pT2 or higher) than in early stage cancer (pT1 or lower) $(\mathrm{P}<0.05)$ (38). This may be due to different tissue processing, different used antibody and the heterogeneity of urinary bladder cancer between both studies,

Previous studies showed that PPARG agonists have been reported to have an inhibitory effect on bladder cancer 
(39, 40 and 41). But, another study showed that PPARG agonist might increase the risk of bladder cancer (42). This discrepancy among studies regarding the role of PPARG in bladder cancer is still unknown, but may be due to different techniques and different clones used. This might also be due to specific pharmacokinetic properties of PPARG ligands or the stage of cancer development at which the PPARG ligand is administered.

As regard the molecular subtype, there was a highly significant statistical correlation between the PPARG expression and the molecular subtype $(\mathrm{P}<0.01)$. The luminal tumours were characterized by the overexpression of PPARG expression parallel goes hand in hand with the study which stated that the "luminal" subtype showed more PPARG expression $(\mathrm{P}=0.004)$ (43). On the other hand, the non-luminal tumours, referred to as basal and double negative subtypes in this study, rarely expressed PPARG $\quad(\mathrm{P}<0.01)$ matched with that study which suggested that the non-luminal tumours expressed low or no PPARG immunostain $(\mathrm{P}<0.05)$ (44).

In our study, the expression of PDL1 was statistically significant with muscle invasion $(\mathrm{P}<0.05)$. Agreeing with this, what was observed that muscle invasive bladder cancer specimens expressed significantly more PD-L1 $(p<0.001)$ (45). Also, it was found that higher levels of PD-L1 expression on tumour cells may promote tumor invasiveness and metastatic potential $(\mathrm{P}=0.004)$ (46). But, disagreeing with this was the study which found that PD-L1 positivity was not different between noninvasive or invasive urothelial bladder cancer $(\mathrm{P}=0.53)$ (47). This may be attributable to the focal nature of PD-L1 expression within tumors, combined with considerable intra-tumour heterogeneity in urothelial bladder cancer.

In this study, there was a high statistically significant correlation between PD-L1 expression and the pathologic $T$ stage $(\mathrm{P}<0.01)$. This is merely in line with what was reported that increasing tumor cell PD-L1 expression predicts localized urothelial carcinoma stage progression, independent of tumor grade $(\mathrm{P}=0.012)$ (48). Moreover, the meta-analysis done before concluded that PD-L1-positivity in solid tumors including urothelial carcinoma was significantly related to the higher stage and poor prognosis $(\mathrm{P}<0.0001)(\mathbf{4 9})$. This is not in a perfect concordance with that study which found no statistically significant differences in tumour stage for patients with PDL1-positive and those with PD-L1-negative tumors $(\mathrm{P}>0.05) \quad$ (50). This may be due to that tissue microarrays 
(TMAs) in their study may capture only a small portion of each tumour; and tumours may heterogeneously express PD-L1 which may lead to overestimation or underestimation of true PD-L1 levels.

In the current work, there was insignificant statistical correlation between PD-L1 expression and tumour grade $(\mathrm{P}>0.05)$. Disagreeing with this what was found that a significant association between PD-L1 expression and WHO grade $(\mathrm{P}<0.001)$ was present (51). This may be due to different grading (WHO G1, 2 and 3), possible tumour heterogeneity among both studies and using monoclonal antibody against human B7-H1 (MIH1, mouse IgG1) which differs from the antibody clone used in our study.

In this study, there was an insignificant correlation between PD-L1 expression and lymphovascular invasion $\quad(\mathrm{P}>0.05)$. Unlikely, it was found that the expression of PD-L1 was associated with more aggressive clinicopathological parameters of bladder cancer like LVI (45). This discrepancy may be due to the rarity of detected LVI in our cases, the different threshold for considering a tumor "PD-L1positive" as well as the IHC assay, the used platform and the antibody clone.

In this study, there was direct highly statistically significant correlation between
IMP3 and PD-L1 identifying their augmented prognostic role.

There was inverse significant statistical correlation between IMP3 and PPARG expressions. This is may be explained by the oncogenic role of IMP3 and the role played by PPARG in urothelial differentiation.

\section{Conclusion}

IMP3 could be used as a prognostic marker as its expression is correlated with adverse prognostic factors. PPARG may be used as a helpful marker for molecular subtyping that can identify luminal urothelial tumours. PD-L1 may be used as a prognostic marker for tumour aggressiveness and so it may be used in immunotherapy. The combination of IMP3 and PD-L1 augment their predictive role in urothelial bladder carcinoma prognosis.

It is recommended that additional wide scale prospective studies using different molecular methods on IMP3, PPARG and PD-L1 are required to clarify their role.

\section{References:}

1.Antoni, S., Ferlay, J., Soerjomataram, I., Znaor, A., Jemal, A., \& Bray, F. (2017): Bladder cancer incidence and mortality: a global overview and recent trends. European urology, 71(1), 96-108.

2.Andreassen, B. K., Grimsrud, T. K., \& Haug, E. S. (2018): Bladder cancer survival: women better off in the long run. European Journal of Cancer, 95, 52-58.

3.He, H., Xie, H., Chen, Y., Li, C., Han, D., Xu, F.,et al., (2020): Global, regional, and national 
burdens of bladder cancer in 2017: estimates from the 2017 Global Burden of Disease study.

4.Ibrahim, A. S., Khaled, H. M., Mikhail, N. N., Baraka, H., \& Kamel, H. (2014): Cancer incidence in Egypt: results of the national population-based cancer registry program. Journal of cancer epidemiology, 2014.

5.Freedman, N. D., Silverman, D. T., Hollenbeck, A. R., Schatzkin, A., \& Abnet, C. C. (2011): Association between smoking and risk of bladder cancer among men and women. Jama, 306(7), 737-745.

6.Cumberbatch, M. G., Cox, A., Teare, D., \& Catto, J. W. (2015): Contemporary occupational carcinogen exposure and bladder cancer: a systematic review and meta-analysis. JAMA oncology, 1(9), 1282-1290.

7.Smith, A. B., Deal, A. M., Woods, M. E., Wallen, E. M., Pruthi, R. S., Chen, R. C., et al., (2014): Muscle-invasive bladder cancer: evaluating treatment and survival in the National Cancer Data Base. BJU international, 114(5), 719-726.

8.Shin, H. J., Lim, S. J and Jeon, H. B. (2018): Pathophysiology of Bladder Cancer. Bladder Cancer, Chapter 3 (35) Pages 33-41.

9.Kluth, L. A., Black, P. C., Bochner, B. H., Catto, J., Lerner, S. P., Stenzl, A., et al., (2015): Prognostic and prediction tools in bladder cancer: a comprehensive review of the literature. European urology, 68(2), 238-253.

10.Cambier, S., Sylvester, R. J., Collette, L., Gontero, P., Brausi, M. A., Van Andel, G., et al., (2016): EORTC nomograms and risk groups for predicting recurrence, progression, and disease-specific and overall survival in nonmuscle-invasive stage Ta-T1 urothelial bladder cancer patients treated with 1-3 years of maintenance bacillus CalmetteGuérin. European urology, 69(1), 60-69.

11.Gong, Y., Woda, B. A., \& Jiang, Z. (2014): Oncofetal protein IMP3, a new cancer biomarker. Advances in anatomic pathology, 21(3), 191-200.

12.Yan, J., Wei, Q., Jian, W., Qiu, B., Wen, J., Liu, J., et al., (2016): IMP3 predicts invasion and prognosis in human lung adenocarcinoma. Lung, 194(1), 137-146.

13.Zhou, Y., Huang, T., Siu, H. L., Wong, C. C., Dong, Y., Wu, F., et al., (2017): IGF2BP3 functions as a potential oncogene and is a crucial target of miR-34a in gastric carcinogenesis. Molecular cancer, 16(1), 1-14.

14.Wei, Q., Zhou, H., Zhong, L., Shi, L., Liu, J., Yang, Q., et al., (2017): IMP3 expression in biopsy specimens as a diagnostic biomarker for colorectal cancer. Human pathology, 64, 137144.

15.Senoo, J., Mikata, R., Kishimoto, T., Hayashi, M., Kusakabe, Y., Yasui, S., et al., (2018): Immunohistochemical analysis of IMP3 and p53 expression in endoscopic ultrasound-guided fine needle aspiration and resected specimens of pancreatic diseases. Pancreatology, 18(2), 176183.

16.Lefterova, M. I., Haakonsson, A. K., Lazar, M. A., \& Mandrup, S. (2014): PPAR $\gamma$ and the global map of adipogenesis and beyond. Trends in Endocrinology \& Metabolism, 25(6), 293302.

17.Lecarpentier, Y., Claes, V., Vallée, A., \& Hébert, J. L. (2017): Thermodynamics in cancers: opposing interactions between PPAR gamma and the canonical WNT/beta-catenin pathway. Clinical and translational medicine, 6(1), 14.

18.Alsaab, H. O., Sau, S., Alzhrani, R., Tatiparti, K., Bhise, K., Kashaw, S. K., et al., (2017): PD1 and PD-L1 checkpoint signaling inhibition for cancer immunotherapy: mechanism, combinations, and clinical outcome. Frontiers in pharmacology, 8, 561 .

19.Zhou, T. C., Sankin, A. I., Porcelli, S. A., Perlin, D. S., Schoenberg, M. P., \& Zang, X. (2017): A review of the PD-1/PD-L1 checkpoint in bladder cancer: From mediator of immune escape to target for treatment1. In Urologic Oncology: Seminars and Original Investigations (Vol. 35, No. 1, pp. 14-20).

20.Hirsch, F. R., McElhinny, A., Stanforth, D., Ranger-Moore, J., Jansson, M., Kulangara, K., et al., (2017): PD-L1 immunohistochemistry assays for lung cancer: results from phase 1 of the blueprint PD-L1 IHC assay comparison project. Journal of Thoracic Oncology, 12(2), 208-222.

21.Compérat, E. M., Burger, M., Gontero, P., Mostafid, A. H., Palou, J., Rouprêt, M., et al., (2018): Grading of Urothelial Carcinoma and The New "World Health Organisation Classification of Tumours of the Urinary System 
and Male Genital Organs 2016”. European Urology Focus (EUF)-435.

22.Wang, G., \& McKenney, J. K. (2018): Urinary

Bladder Pathology: World Health Organization (WHO) Classification and American Joint Committee on Cancer (AJCC) Staging Update. Archives of pathology \& laboratory medicine.

23.Dadhania, V., Zhang, M., Zhang, L., Bondaruk, J., Majewski, T., Siefker-Radtke, A., et al., (2016): Meta-analysis of the luminal and basal subtypes of bladder cancer and the identification of signature immunohistochemical markers for clinical use. EBioMedicine, 12, 105-117.

24.Abd ElAziz, T., Amer, R., Bassyoni, O., \& Ahmed, H. (2020). Significance of Insluin-Like Growth Factor II mRNA Binding Protein 3 (IMP3) Expression In Selected Thyroid Lesions. Benha Medical Journal, 37(2), 462476.

25.Mylona, E., Giannopoulou, I., Diamantopoulou, K., Bakarakos, P., Nomikos, A., Zervas, A., et al., (2009): Peroxisome proliferator-activated receptor gamma expression in urothelial carcinomas of the bladder: association with differentiation, proliferation and clinical outcome. European Journal of Surgical Oncology (EJSO), 35(2), 197-201.

26.Phillips, T., Simmons, P., Inzunza, H. D., Cogswell, J., Novotny Jr, J., Taylor, C., et al., (2015). Development of an automated PD-L1 immunohistochemistry (IHC) assay for nonsmall cell lung cancer. Applied immunohistochemistry \& molecular morphology, 23(8), 541.

27.Wachter, D. L., Schlabrakowski, A., Hoegel, J., Kristiansen, G., Hartmann, A., \& Riener, M. O. (2011): Diagnostic value of immunohistochemical IMP3 expression in core needle biopsies of pancreatic ductal adenocarcinoma. The American journal of surgical pathology, 35(6), 873-877.

28.Sandes, E. O., Lodillinsky, C., Langle, Y., Belgorosky, D., Marino, L., Gimenez, L., et al., (2012): Inducible nitric oxide synthase and $\operatorname{PPAR} \gamma$ are involved in bladder cancer progression. The Journal of urology, 188(3), 967-973.

29.US FDA (2019): limits the use of Tecentriq and Keytruda for some urothelial cancer patients.
Available from: https://www.fda.gov/drugs/infor mationondrugs/approveddrugs/ucm612484.htm

30.Cimadamore, A., Scarpelli, M., Massari, F., Eckstein, M., Gevaert, T., Cheng, L., et al., (2020): Immunotherapy for urothelial cancer: from the diagnostic pathologist's point of view.

31.Yang, F., Zhou, Q., Meng, L., \& Xing, N. (2019): IMP3 is a biomarker for non-muscleinvasive urothelial carcinoma of the bladder associated with an aggressive phenotype. Medicine, 98(27).

32.Burdelski, C., Jakani-Karimi, N., Jacobsen, F., Möller-Koop, C., Minner, S., Simon, R., et al., (2018): IMP3 overexpression occurs in various important cancer types and is linked to aggressive tumor features: A tissue microarray study on 8,877 human cancers and normal tissues. Oncology reports, 39(1), 3-12.

33.Chen, L., Xie, Y., Li, X., Gu, L., Gao, Y., Tang, L., et al., (2017): Prognostic value of high iMP3 expression in solid tumors: a metaanalysis. OncoTargets and therapy, 10, 2849.

34.Xylinas, E., Cha, E. K., Khani, F., Kluth, L. A., Rieken, M., Volkmer, B. G., et al.,. (2014): Association of oncofetal protein expression with clinical outcomes in patients with urothelial carcinoma of the bladder. The Journal of urology, 191(3), 830-841.

35.Kim , K., Sung, C. O., Park, B. H., Ku, J. Y., Go, H., Ro, J. Y., et al., (2015): Immunoprofilebased subgrouping of urothelial bladder carcinomas for survival prediction. Human pathology, 46(10), 1464-1470.

36.Korpal, M., Puyang, X., Wu, Z. J., Seiler, R., Furman, C., Oo, H. Z., et al., (2017): Evasion of immunosurveillance by genomic alterations of PPAR $\gamma /$ RXR $\alpha$ in bladder cancer. Nature communications, 8(1), 1-14.

37.Zhang, Z., Xu, H., Ji, J., Shi, X., Lyu, J., Zhu, Y., et al., (2019): Heterogeneity of PTEN and PPAR- $\gamma$ in cancer and their prognostic application to bladder cancer. Experimental and Therapeutic Medicine, 18(4), 3177-3183.

38.Yoshimura, R., Matsuyama, M., Segawa, Y., Hase, T., Mitsuhashi, M., Tsuchida, K., et al., (2003): Expression of peroxisome proliferatoractivated receptors (PPARs) in human urinary bladder carcinoma and growth inhibition by its agonists. International journal of cancer, 104(5), 597-602. 
39.Kassouf, W., Chintharlapalli, S., Abdelrahim, M., Nelkin, G., Safe, S., \& Kamat, A. M. (2006): Inhibition of bladder tumor growth by 1 , 1-bis (3'-indolyl)-1-(p-substitutedphenyl) methanes: a new class of peroxisome proliferator-activated receptor $\gamma$ agonists. Cancer research, 66(1), 412-418.

40.Wang, G., Cao, R., Wang, Y., Qian, G., Dan, H. C., Jiang, W., et al., (2016): Simvastatin induces cell cycle arrest and inhibits proliferation of bladder cancer cells via PPAR $\gamma$ signalling pathway. Scientific reports, 6, 35783.

41.Cao, R., Wang, G., Qian, K., Chen, L., Ju, L., Qian, G., et al., (2018): TM4SF1 regulates apoptosis, cell cycle and ROS metabolism via the PPAR $\gamma$-SIRT1 feedback loop in human bladder cancer cells. Cancer letters, 414, 278293.

42.Tang, H., Shi, W., Fu, S., Wang, T., Zhai, S., Song, Y., et al., (2018): Pioglitazone and bladder cancer risk: a systematic review and meta-analysis. Cancer medicine, 7(4), 10701080.

43.Choi, W., Porten, S., Kim, S., Willis, D., Plimack, E. R., Hoffman-Censits, J., et al., (2014): Identification of distinct basal and luminal subtypes of muscle-invasive bladder cancer with different sensitivities to frontline chemotherapy. Cancer cell, 25(2), 152-165.

44.Sjödahl, G., Jackson, C. L., Bartlett, J. M., Siemens, D. R., \& Berman, D. M. (2019): Molecular profiling in muscle-invasive bladder cancer: more than the sum of its parts. The Journal of pathology, 247(5), 563-573.

45.Huang, Y., Zhang, S. D., McCrudden, C., Chan, K. W., Lin, Y., \& Kwok, H. F. (2015): The prognostic significance of PD-L1 in bladder cancer. Oncology Reports, 33(6), 3075-3084.

46.Bardoli, A. D., Afshar, M., Viney, R., Foster, M., Porfiri, E., Zarkar, A., et al., (2016): The PD-1/PD-L1 axis in the pathogenesis of urothelial bladder cancer and evaluating its potential as a therapeutic target.

47. Bellmunt, J., Mullane, S. A., Werner, L., Fay, A. P., Callea, M., Leow, J. J., et al., (2015): Association of PD-L1 expression on tumorinfiltrating mononuclear cells and overall survival in patients with urothelial carcinoma. Annals of oncology, 26(4), 812-817.

48. Inman, B. A., Sebo, T. J., Frigola, X., Dong, H., Bergstralh, E. J., Frank, I., et al., (2007): PD-L1 (B7-H1) expression by urothelial carcinoma of the bladder and BCG-induced granulomata: associations with localized stage progression. Cancer: Interdisciplinary International Journal of the American Cancer Society, 109(8), 1499-1505.

49.Wang, Q., Liu, F., \& Liu, L. (2017): Prognostic significance of PD-L1 in solid tumor: an updated meta-analysis. Medicine, 96(18).

50.Davick, J. J., Frierson, H. F., Smolkin, M., \& Gru, A. A. (2018): PD-L1 expression in tumor cells and the immunologic milieu of bladder carcinomas: a pathologic review of 165 cases. Human pathology, 81, 184-191.

51. Nakanishi, J., Wada, Y., Matsumoto, K., Azuma, M., Kikuchi, K., \& Ueda, S. (2007): Overexpression of B7-H1 (PD-L1) significantly associates with tumor grade and postoperative prognosis in human urothelial cancers. Cancer Immunology, Immunotherapy, 56(8), 11731182.

To cite this article: Rasha M. El Sawi, Ebtehal Abdel Aal , Amr El-Sebaaie, Nehal Zafer, Hala Agina. Significance of Expression of IMP3, PPARG \& PD-L1 in Urothelial Carcinoma (Immunohistochemical Study) BMF 2021; 38(academic issue): 112- 127. DOI: $10.21608 / \mathrm{bmfj} .2020 .130876$ 